\title{
Commentary \\ Is bronchoalveolar lavage with quantitative cultures a useful tool for diagnosing ventilator-associated pneumonia?
}

\author{
Jean-Yves Fagon ${ }^{1}$, Jean Chastre ${ }^{2}$ and Jean-Jacques Rouby ${ }^{3}$
}

\begin{abstract}
${ }^{1}$ Réanimation Médicale, Hôpital Européen Georges Pompidou, Assistance Publique-Hôpitaux de Paris, Université Paris-Descartes, Paris, France ${ }^{2}$ Réanimation Médicale, Groupe Hospitalier Pitié-Salpêtrière, Assistance Publique-Hôpitaux de Paris, Université Pierre et Marie Curie of Paris-6, France ${ }^{3}$ Réanimation Chirurgicale, Groupe Hospitalier Pitié-Salpêtrière, Assistance Publique-Hôpitaux de Paris, Université Pierre et Marie Curie of Paris-6, France
\end{abstract}

Corresponding author: Jean-Yves Fagon, jean-yves.fagon@egp.aphp.fr

Published: 16 April 2007

This article is online at http://ccforum.com/content/11/2/123

(c) 2007 BioMed Central Ltd
Critical Care 2007, 11:123 (doi:10.1186/cc5724)

with VAP, but also improving our ability to diagnose and exclude infection in the intensive care unit (ICU) setting in order to avoid administering antibiotics to patients without bacterial infection [4].

This recent published randomized trial [1] comparing the quantitative culture of bronchoalveolar lavage (BAL) fluid and the culture of endotracheal aspirate in critically ill patients with suspected VAP adds to the information presented by four previous trials [5-8]. The Canadian Critical Care Trials Group found that the two diagnostic techniques were associated with similar clinical outcomes and similar overall use of antibiotics (Table 1). Several considerations should be taken into account, however, to appropriately evaluate the possible impact of diagnostic techniques on the individual (patient morbidity and mortality) and on the collective (emergence and dissemination of antibiotic-resistant strains) outcomes.

First, as clearly underlined by Kollef in his related editorial [9], the exclusion of patients previously colonized or infected with methicillin-resistant Staphylococcus aureus or Pseudomonas species and the exclusion of those patients having previously received the 'study drugs' (that is, meropenem and/or ciprofloxacin) resulted in a low rate of studied patients with 'high-risk' pathogens responsible for VAP. A proportion of less than $12 \%$ of difficult-to-treat pathogens, such as $P$. aeruginosa, Acinetobacter spp., Stenotrophomonas maltophilia, and/or methicillin-resistant $S$. aureus, as compared with more than $30 \%$ in the French study [8], diminishes the usefulness of the results of this study in real life.

Second, 29\% of patients managed using BAL had new antibiotics initiated within 3 days before randomization, probably after the onset of the first signs in relation to VAP,

$\mathrm{BAL}=$ bronchoalveolar lavage $; \mathrm{ICU}=$ intensive care $u$ it $; \mathrm{VAP}=$ ventilator-associated pneumonia 


\begin{tabular}{|c|c|c|}
\hline & $\begin{array}{l}\text { Endotracheal aspiration } \\
\qquad(n=374)\end{array}$ & $\begin{array}{l}\text { Bronchoalveolar lavage } \\
\qquad(n=365)\end{array}$ \\
\hline \multicolumn{3}{|l|}{ Outcomes } \\
\hline Mortality at day $28(\%)$ & 18.4 & 18.9 \\
\hline Duration of mechanical ventilation (days) & $8.8(7.0-10.7)$ & $8.9(7.4-10.7)$ \\
\hline Duration of intensive care unit stay (days) & $12.2(10.9-14.2)$ & $12.3(10.9-13.8)$ \\
\hline Final diagnosis of ventilator-associated pneumonia (\%) & 82.9 & 86.3 \\
\hline \multicolumn{3}{|l|}{ Antibiotics } \\
\hline Adequacy of empirical treatment among patients with positive cultures (\%) & 89.5 & 89.0 \\
\hline Targeted therapy by day $6(\%)$ & 74.6 & 74.2 \\
\hline Number of days alive without antibiotics & $10.4 \pm 7.5$ & $10.6 \pm 7.9$ \\
\hline
\end{tabular}

No differences were statistically significant.

which is problematic when using quantitative culture techniques. In this case, a negative finding or a result below the usual threshold of $10^{4}$ colony-forming units $/ \mathrm{ml}$ could indicate either that the patient has been successfully treated for pneumonia and the bacteria are eradicated, or that there was no lung infection to begin with [10]. These authors did not give any information on how decisions regarding antibiotic treatment were taken in this group of patients.

Third, the authors report a relatively high rate (14\%) of inappropriate initial empirical therapy in the BAL group. As indicated above, the low frequency of high-risk, difficult-totreat pathogens responsible for pneumonia cannot explain such a disappointing result, when compared with the $0.5 \%$ rate of inappropriate initial therapy reported by Fagon and coworkers in the invasive strategy group [8]. The most probable explanation is that all patients included in this study were also randomized to receive a fixed combination therapy or monotherapy as initial treatment: meropenem plus ciprofloxacin or meropenem alone. Several studies have clearly established that initial antimicrobial therapy in patients with VAP should be customized to local epidemiology at the ICU level [11].

Fourth, even on day 6 the rate of targeted therapy was only $74.2 \%$ in the BAL arm, underlining the fact that, in many patients managed using this diagnostic technique, early deescalation was not performed although clearly indicated. Unfortunately, information on how decision algorithms were followed in the two study arms once cultures were available (as soon as day 2 or day 3) was not given. Obviously, the potential benefit of using a diagnostic tool such as BAL for safely restricting unnecessary antimicrobial therapy in such a setting can only be obtained when decisions regarding antibiotics are closely linked to bacteriological - both direct examination and cultures - results [12]. In the current study,
BAL was not used for identifying patients with VAP who needed antimicrobial therapy; this decision was essentially left to the ICU physicians in charge of the included patients on the basis of their clinical judgment, even when BAL culture results were $<10^{4}$ colony-forming units $/ \mathrm{ml}$. Interestingly, the proportion of 'confirmed pneumonia' was $86 \%$ in the BAL group and $83 \%$ in the endotracheal aspirate group. In contrast to previous recommendations concerning the use of quantitative $\mathrm{BAL}$, therefore, many patients with quantitative culture results below the cut-off point of $10^{4}$ colony-forming units $/ \mathrm{ml}$ continued to receive antibiotics, even after day 3 . This could entirely explain why there was a similar use of antibiotics in the two study arms.

Finally, a major benefit of a negative BAL specimen may be to direct attention away from the lungs as the source of fever and, in the absence of antibiotic interference, to more readily diagnose other potential sites of infection. Delaying diagnosis or definitive treatment of the true site of infection may lead to prolonged antibiotic therapy and to induction of additional dysfunction $[13,14]$. In the current trial, we are left with uncertainties regarding the numbers of extrapulmonary infection in the two arms of the trial, as well as how long the recommended duration of therapy in patients with VAP should be and the how patients were managed in case of a second episode.

In summary, even if the results of the Canadian study are consistent with those of the three Spanish trials (Table 2) in which antimicrobial treatment was also initiated in all suspected patients and rarely withheld in patients with negative cultures, our own bias is that additional studies will be needed before one can conclude that a strategy based on the systematic collection of distal pulmonary secretions prior to the introduction of new antibiotics and quantitative culture techniques is useless. In real life, the key issue is to be able to 
Table 2

\begin{tabular}{|c|c|c|c|c|c|}
\hline \multirow[b]{2}{*}{ Study } & \multirow[b]{2}{*}{ Sample size } & \multicolumn{2}{|c|}{ 28-day mortality (\%) } & \multicolumn{2}{|c|}{ Antibiotic usage } \\
\hline & & Invasive arm & Clinical arm & Invasive arm & Clinical arm \\
\hline Sanchez-Nieto et al. [5] & 51 & 45.8 & 26.7 & ND & ND \\
\hline Ruiz et al. [6] & 76 & 37.8 & 46.1 & $13 \pm 4^{a}$ & $12 \pm 4$ \\
\hline Sole Violan et al. [7] & 88 & 22.2 & 20.9 & ND & ND \\
\hline Fagon et al. [8] & 413 & 30.9 & 38.8 & $11.5 \pm 9.0^{b}$ & $7.5 \pm 7.6$ \\
\hline Canadian Critical Care Trials Group [1] & 739 & 18.9 & 18.4 & $10.4 \pm 7.5^{c}$ & $10.6 \pm 7.9$ \\
\hline
\end{tabular}

$\mathrm{ND}$, not determined. ${ }^{\mathrm{a}}$ Ruiz et al. [6] reported the total duration of antibiotic treatment; $p=0.48$. ${ }^{\mathrm{b}}$ Fagon et al. [8] reported antibiotic-free days; $p<0.002$. ${ }^{c}$ The Canadian Critical Care Trials Group [1] reported antibiotic-free days; $p=0.86$.

adhere to a de-escalation strategy, which is the only way to curb the unnecessary use of antibiotics in the ICU. The predominant impact of pretest opinion and the absence of clear bacteriological-based decision algorithms in the current study may unfortunately encourage physicians to pursue antibiotics in most patients after 2 days, even once results of bacterial cultures are available.

\section{Competing interests}

The authors declare that they have no competing interests.

\section{References}

1. Canadian Critical Care Trials Group: A randomized trial of diagnostic techniques for ventilator-associated pneumonia. N Engl $J$ Med 2006, 355:2619-2630.

2. Niederman MS, Torres A, Summer W: Invasive diagnostic testing is not needed routinely to manage suspected ventilator-associated pneumonia. Am J Respir Crit Care Med 1994, 150:565-569.

3. Chastre J, Fagon JY: Invasive diagnostic testing should be routinely used to manage ventilated patients with suspected pneumonia. Am J Respir Crit Care Med 1994, 150:570-574.

4. Chastre J, Fagon JY: Ventilator-associated pneumonia. Am J Respir Crit Care Med 2002, 165:867-903.

5. Sanchez-Nieto JM, Torres A, Garcia-Cordoba F, El-Ebiary M, Carrillo A, Ruiz J, Nunez ML, Niederman M: Impact of invasive and noninvasive quantitative culture sampling on outcome of ventilator-associated pneumonia: a pilot study. Am J Respir Crit Care Med 1998, 157:371-376.

6. Ruiz M, Torres A, Ewig S, Marcos MA, Alcon A, Lledo R, Asenjo MA, Maldonaldo A: Noninvasive versus invasive microbial investigation in ventilator-associated pneumonia: evaluation of outcome. Am J Respir Crit Care Med 2000, 162:119-125.

7. Sole Violan J, Fernandez JA, Benitez AB, Cardenosa Cendrero JA, Rodriguez de Castro F: Impact of quantitative invasive diagnostic techniques in the management and outcome of mechanically ventilated patients with suspected pneumonia. Crit Care Med 2000, 28:2737-2741.

8. Fagon JY, Chastre J, Wolff M, Gervais C, Parer-Aubas S, Stephan F, Similowski T, Mercat A, Diehl JL, Sollet JP, Tenaillon A: Invasive and noninvasive strategies for management of suspected ventilator-associated pneumonia. A randomized trial. Ann Intern Med 2000, 132:621-630.

9. Kollef $\mathrm{M}$ : Diagnosis of ventilator-associated pneumonia. $N$ Engl J Med 2006, 355:2691-2693.

10. Souweine B, Veber B, Bedos JP, Gachot B, Dombret MC Regnier B, Wolff M: Diagnostic accuracy of protected specimen brush and bronchoalveolar lavage in nosocomial pneumonia: impact of previous antimicrobial treatment. Crit Care Med 1998, 26:236-244.

11. Rello J, Sa-Borges M, Correa H, Leal SR, Baraibar J: Variations in etiology of ventilator-associated pneumonia across four treatment sites: implications for antimicrobial prescribing practices. Am J Respir Crit Care Med 1999, 160:608-613.

12. American Thoracic Society, Infectious Diseases Society of America: Guidelines for the management of adults with hospital-acquired, ventilator-associated, and healthcare-associated pneumonia. Am J Respir Crit Care Med 2005, 171:388-416.

13. Meduri GU, Mauldin GL, Wunderink RG, Leeper KV, Jr, Jones CB, Tolley E, Mayhall G: Causes of fever and pulmonary densities in patients with clinical manifestations of ventilator-associated pneumonia. Chest 1994, 106:221-235.

14. Liu YC, Huang WK, Huang TS, Kunin CM: Inappropriate use of antibiotics and the risk for delayed admission and masked diagnosis of infectious diseases: a lesson from Taiwan. Arch Intern Med 2001, 161:2366-2370. 